\title{
Examining the relationship between global and domain measures of quality of life by three factor structure models
}

\author{
Chia-Huei Wu • Grace Yao
}

Received: 17 November 2006/ Accepted: 18 December 2006/Published online: 26 January 2007

(C) Springer Science+Business Media B.V. 2007

\begin{abstract}
This study examined the relationship between global and domain measures of quality of life from a psychometric perspective by three different factor structure models. Three hundred and four students at National Taiwan University participated in this study. They completed the Satisfaction with Life Scale (SWLS, a global measurement for quality of life) and the WHOQOL-BREF (a domain-specific measurement for quality of life). Three models were specified to examine the relationships among scores of the SWLS and the WHOQOL-BREF. The first model was a common factor model in which scores of the SWLS and the WHOQOL-BREF were all influenced by a single factor. The second model was a correlated two-factor model in which scores of the SWLS were influenced by one factor and scores of the WHOQOL-BREF influenced by another factor, with these two factors being allowed to be correlated. The third model was a three-factor model, in which one factor (representing quality of life) influenced the scores of the SWLS and the WHOQOL-BREF, another factor (representing global approach) only influenced the scores of the SWLS, and yet another factor (representing domain approach) only influenced the scores of the WHOQOL-BREF. The results showed that the third model was the best, suggesting that global measures (the SWLS) and domain measures (the WHOQOL-BREF) did assess the same construct on quality of life, however, the measurement approaches they adopted (global or domain approach) also have substantial impact on the meaning of scores.
\end{abstract}

Keywords Global · Domain-specific · Quality of life · Measurement · Confirmatory factor analysis

C.-H. Wu · G. Yao (ه)

Department of Psychology, National Taiwan University, 1, Sec. 4, Roosevelt RD., Taipei, Taiwan, ROC

e-mail: kaiping@ntu.edu.tw 


\section{Introduction}

It is well known that measurements for quality of life can be divided into two main categories, that is, global measurements from a top-down perspective and domainspecific measurements from a bottom-up perspective. A top-down perspective regards evaluations in various life domains as resulting from evaluation of life as a whole (Diener, 1984; Diener, Suh, Lucas, \& Smith 1999; Lance, Mallard, \& Michalos, 1995). Based on this notion, it is meaningless to assess individuals' quality of life by several specific domains. Accordingly, global measurements are developed and respondents are usually asked to evaluate their life as a whole based on these measurements. Because the global evaluation of life is related to a stable propensity for an individual, the advantage of global measurements is to facilitate us to assess and compare the individual differences on the quality of life on a single indicator.

Conversely, a bottom-up perspective regards evaluations in various life domains as contributing to the evaluation of life as a whole (Diener, 1984; Diener et al., 1999; Lance et al., 1995). Based on this notion, one should assess individuals' life quality in various life domains. Therefore, domain-specific measurements are developed to measure life quality in several specific domains. Respondents are usually asked to evaluate their life in these specific life domains, and then, several domain scores are used to indicate respondents' quality of life status. The advantage of domain-specific measurements is that they can exhibit performance of each life domain, and based on the information collected enable researchers to give advice to or intervention for each respondent.

It is obvious that the concerns of global measurements and domain-specific measurements are different, although both are aimed at measuring individuals' quality of life. In this study, it should be noted that quality of life is a broad term referring to individuals' subjective evaluation of their lives. However, the question of how to characterize the relationship between global and domain-specific measures on quality of life analysis remains. In the existing literature, the relationship between global evaluation and domain-specific evaluations has been investigated from different perspectives. Some researchers have addressed this issue from a theoretical debate of both top-down and bottom-up perspectives. For example, Lance et al. (1995) tested alternative theoretical models that specified bottom-up, top-down, and bidirectional relationships between overall life satisfaction and satisfaction with 11 life facets using several structural equation models. Their results showed that the bidirectional model was the best model, suggesting a non-recursive relationship between global measure and domain-specific measures.

Moreover, some researchers have dealt with the same issue by examining the relationships among global measure and domain-specific measures using the multidimensional scaling (MDS) technique. For example, Cohen (2000) measured satisfaction and have-want discrepancy evaluations in 11 life facets and overall life satisfaction for each participant. Then, a correlation matrix among satisfaction scores for 11 life facets and overall life was analyzed with MDS. The result showed that satisfaction with overall life located in the center of the MDS configuration as a point of origin and satisfaction with 11 life facets were radiated around the satisfaction with overall life. A similar result was gained from have-want discrepancy measures for 11 life facets and overall life. This finding implied that evaluations on various life domains will merge into the overall life satisfaction. 
Furthermore, other researchers have discussed the global-domain measurement issue from a weighting approach to see if global evaluation is made by weighting domain-specific evaluations (e.g., Hsieh, 2003, 2004; Russell, Hubley, Palepu, \& Zumbo, 2006; Wu \& Yao, 2006a, b). Researchers in this line of study usually strive to find a weighting theme underlying specific life domains for forming a global evaluation of life. Although the idea of weighting seems reasonable, Hsieh (2003), Russell et al. (2006) and $\mathrm{Wu}$ and Yao (2006a) found that weighted scores did not perform better than unweighted scores in predicting global life evaluation. These empirical findings did not support the notion that global evaluation is made by weighting domain-specific evaluations.

In summary, all the previous studies treated global and domain-specific measures as two distinct constructs, implicitly assuming that global and domain-specific measures are different constructs (although both of them were aimed to assess the concept of QOL). Thus, it is an interesting issue to investigate if global and domainspecific measures represented the same or different constructs of QOL. If they are the same constructs, then, analyzing the relationship between global and domainspecific measures by bidirectional model (Lance et al., 1995), MDS (Cohen, 2000), and importance-weighting approach (e.g., Hsieh, 2003, 2004; Wu \& Yao, 2006a, b) would not have any merit. Therefore, this study was aimed to discuss the global and domain measurement issue by examining the factor structure of a global measurement and a domain-specific measurement. Although the technique of factor analysis is not a new method in the literature, however, it deserved to be applied to the issue of global-domain measurements, because it can facilitate us to examine if these two kinds of measurements tap distinct meanings of quality of life.

In order to examine the relationship between global and domain measurements, two measurements were selected. For the global measurement, the Satisfaction with Life Scale (SWLS) (Diener, Emmons, Larsen, \& Griffin, 1985) was used. This scale has been widely used in empirical QOL studies. This scale contains 5 items measuring individuals' satisfaction with life as a whole. The factor structure of this scale is one common factor for the 5 items. For the domain-specific measurement, the WHOQOL-BREF adapted to Taiwan was used (Yao, Chung, Yu, \& Wang, 2002). The WHOQOL-BREF was developed by the World Health Organization (The WHOQOL Group, 1998a) from WHOQOL-100 (The WHOQOL Group, 1998b). It contains 26 items in which 24 items were designed to measure quality of life in four domains, including physical health, psychological health, social relationships, and environmental health. The factor structure of the WHOQOL-BREF at domain level was one common factor underlying the four domain scores (the full structure for the WHOQOL-BREF was a hierarchal factor structure in which there is a second-order factor and four first-order factors underlying the 24 items).

In this study, the factor structures of these two measurements were examined in different models to address the issue of global-domain measurement. There were three confirmatory-factor-analysis (CFA) models specified to address this issue. The first model was a common factor model specified for the SWLS and the WHOQOL-BREF. This model assumed that global and domain measures tapped the same construct for quality of life, regardless of whether a global or domain approach was being used.

The second model was a correlated two-factor model for the SWLS and the WHOQOL-BREF. This model assumed that the SWLS and the WHOQOL-BREF tapped different but related constructs. That is, measurements adopting global or 
domain approach tapped different constructs of what they measured, although these two constructs were allowed to be correlated.

The third model was a three-factor model. There was one common factor (representing quality of life) for both the SWLS and the WHOQOL-BREF, one factor (representing global approach) for the SWLS, and another factor (representing domain approach) for the WHOQOL-BREF. This model assumed that both the SWLS and the WHOQOL-BREF tapped the same construct on quality of life, however, the global or domain approach they used may have its impact in the factor structure. This three-factor model can help us differentiate the influence of the quality of life construct and the influence of the measurement approach on the observed scores.

Generally, the three models proposed above were fit to empirical data by CFA to see which was better in describing the relationships among global and domain measures.

\section{Method}

\subsection{Participants}

Three hundred and four students at National Taiwan University participated in this study. The sample consisted of $38.2 \%$ male $(n=116)$ and $61.8 \%$ female $(n=188)$ respondents. The mean age was 20.1 years (standard deviation $=1.67$, ranged from 17 to 27). All questionnaires were filled out in approximately $20 \mathrm{~min}$ and then handed in to the investigator.

\subsection{Instruments}

\subsubsection{Satisfaction with Life Scale}

The SWLS was developed by Diener et al. (1985). It contains 5 items and employs a 7-point Likert-type scale with higher values corresponding to a higher degree of satisfaction. The SWLS showed good reliability and validity. The internal reliability ranged from .79 to .89 . The test-retest reliability coefficients of the SWLS were .83 for a 2-week interval, .84 for a 1-month interval, .64 and .82 for a 2-month interval in different studies (see Pavot \& Diener, 1993). In addition, it has shown that the SWLS has only one factor and this single-factor solution has been replicated in different studies (Arrindell, Heesink, \& Feij, 1999; Arrindell, Meeuwesen, \& Huyse, 1991; Atienza, Balaguer, \& Garcia-Merita, 2003; Diener et al., 1985; Lewis et al., 1995; Pavot et al., 1991; Shevlin, Brunsden, \& Miles, 1998; Shevlin \& Bunting, 1994; Westaway, Maritz, \& Golele, 2003). Regarding validity, the SWLS demonstrated adequate construct validity, convergent validity, and discriminant validity (see Arrindell et al., 1999; Lucas, Diener, \& Suh, 1996; Pavot \& Diener, 1993). In a Chinese translation of the scale published in Hong Kong, Sachs (2003) showed a single factor underlying the scale and demonstrated it's good validity. In addition, Wu and Yao's study (2006c) confirmed the single-factor structure of the SWLSTaiwan version, and revealed the SWLS-Taiwan version was factor invariant across gender. In this study, the internal consistency (coefficient $\alpha$ ) of the scale was .90 . 


\subsubsection{The WHOQOL-BREF questionnaire}

The WHOQOL-BREF version was administered in this study. It was developed from the WHOQOL-100 version and contains four domains related to quality of life (physical health, psychological health, social relationships, and environmental health), as well as one facet measuring overall quality of life and general health. It has 26 standard items from the WHOQOL-BREF. The 26 standard items were taken from each of the 24 facets contained in the WHOQOL-100, and two from the Overall Quality of Life and General Health facet in the WHOQOL-100. In Yao et al.'s (2002) study, exploratory and confirmatory factor analyses of the WHOQOL-BREF Taiwan version revealed a four-factor model (physical, psychological, social and environmental factors). The internal consistency (Cronbach's alpha) coefficients ranged from .70 to .77 for the four domains (.73 to .83 in this study). The test-retest reliability coefficients with 2-4 weeks interval ranged from .41 to .79 at item/facet level and .76 to .80 at domain level (all $p<.01$ ). Content validity coefficients were in the range of .53 to .78 for item-domain correlations and .51 to .64 for inter-domain correlations (all $p<.01$ ). The four domain scores (physical, psychological, social, and environment domains) ranging from 4 to 20 were computed by the standard scoring algorithms of the WHOQOL-BREF version. The two items from the Overall Quality of Life and General Health facet in the WHOQOL-100 were not included in these four domain scores.

\subsection{Model specification and evaluation}

Three models were examined in the current research. The first model was a common factor model specified for the SWLS and the WHOQOL-BREF. That is, the five item scores in the SWLS and the four domain scores in the WHOQOL-BREF were influenced by the same factor. In addition, factor variance was set as 1 and uniqueness (error) for each score was uncorrelated in this model. Therefore, this model estimated 18 parameters with 45 data points that resulted in 27 degrees of freedom.

The second model was a correlated two-factor model for the SWLS and the WHOQOL-BREF. In this model, there was a factor influencing the five item scores in the SWLS and a factor influencing the four domain scores in the WHOQOLBREF. In addition, the two factors were correlated. Like the first model, factor variances were set as 1 and uniqueness (error) for each score was uncorrelated in this one. This model estimated 19 parameters with 45 data points that resulted in 26 degrees of freedom.

The third model was a three-factor model. There was one common factor, representing quality of life, for both the SWLS and the WHOQOL-BREF; another factor, representing global approach, for the SWLS; and yet another factor, representing domain approach, for the WHOQOL-BREF. Like the first two models, factor variances were set as 1 and uniqueness (error) for each score was uncorrelated in this model. In order to test if domain and global approaches are independent constructs, factors for domain and global approaches were specified to be correlated to see if their correlation was significant. This model estimated 28 parameters with 45 data points that resulted in 17 degrees of freedom. 
Regarding model evaluation, different information was considered in making a general conclusion. First, for overall model fit, four fit indices were used in conjunction with Chi-square tests in evaluating the model according to $\mathrm{Hu}$ and Bentler's (1999) suggestion. Two incremental fit indices, the non-normed fit index (NNFI) and the comparative fit index (CFI), were chosen for use in this study. A value of NNFI and CFI exceeding .95 indicates a good fit, while a value between .90 and .95 represents an adequate fit for a model (Bentler, 1990; Hoyle, 1995). Recently, Hu and Bentler (1999) suggested a more stringent cutoff of .95 or above on the NNFI and CFI. In addition, two absolute fit indices, the standardized root mean squared residual (SRMR) and the root mean squared error of approximation (RMSEA), were also used. For RMSEA, a well-fitting model should be at or below .05 and at or below .08 for a model with reasonable fit (Browne \& Cudeck, 1993). Hu and Bentler (1999) suggested a cutoff value of .06 for a well-fitting model. For SRMR, Hu and Bentler (1999) suggested SRMR should be at .08 or less for a good fit. In addition, they also recommended using a cutoff value close to .95 for CFI in combination with a cutoff value close to .09 for SRMR to evaluate model fit $(\mathrm{CFI} \geq .95 ; \mathrm{SRMR} \leq .09)$.

However, Hu and Bentler (1999) indicated that the rules they proposed should not be over-generalized, because these rules were based on simulation results of models in certain conditions. Indeed, recent simulation studies found that the rules proposed by $\mathrm{Hu}$ and Bentler (1999) cannot be generalized to models in other conditions (Beauducel \& Wittmann, 2005; Fan \& Sivo, 2005; Marsh, Hau, \& Wen 2004; Yuan, 2005). Moreover, Marsh et al. (2004) further pointed that there were logical problems underlying the rationale of a hypothesis-testing approach to setting cutoff vales for fit indices proposed by $\mathrm{Hu}$ and Bentler (1999). In other words, it was inappropriate to use fit indices in hypothesis-testing. Therefore, in the current study, rules proposed by $\mathrm{Hu}$ and Bentler (1999) were only for reference, we did not stick to the criteria they set in model evaluation. In addition to overall model fit, parameter estimates were also examined to see if there is any improper solution, such as unexpected sign or extreme value. Squared multiple correlation was used to evaluate the variance accounted in the equation for each indicator.

Furthermore, because the main purpose of this study was to compare the three models, the most important part in model evaluation is to compare the values of Akaike's information criterion (AIC), Consistent Akaike Information Criterion (CAIC) and Expected Cross-Validation Index (ECVI) (indices for model comparison) among the three models. A model with lower AIC, CAIC and ECVI values represented a better model. All information was considered to make a general conclusion in model evaluation and comparison in this study.

\section{Results}

\subsection{Descriptive statistics}

The descriptive statistics including the mean, standard deviation, skewness, and kurtosis of each score for the five item scores in the SWLS and the four domain scores in the WHOQOL-BREF are presented in Table 1. 
Table 1 Descriptive statistics for scores of the SWLS and the WHOQOL-BREF

\begin{tabular}{lcccc}
\hline & Mean & Std & Skewness & Kurtosis \\
\hline SWLS & & & & \\
Item 1 & 4.11 & 1.40 & -0.20 & -0.67 \\
Item 2 & 4.08 & 1.41 & -0.02 & -0.54 \\
Item 3 & 4.46 & 1.41 & -0.37 & -0.41 \\
Item 4 & 4.51 & 1.48 & -0.32 & -0.56 \\
Item 5 & 4.13 & 1.83 & -0.10 & -1.17 \\
WHOQOL-BREF & & & & 0.16 \\
Physical domain & 12.79 & 2.02 & -0.07 & 0.13 \\
Psychological domain & 12.68 & 2.40 & -0.51 & -0.01 \\
Social domain & 13.67 & 2.68 & -0.29 & 0.33 \\
Environmental domain & 13.38 & 1.97 & -0.25 & \\
\hline
\end{tabular}

\subsection{Confirmatory factor analysis for three models}

In this section, the three models proposed in the current study are examined by confirmatory factor analysis. Because the data did not depart far from normal distribution, the maximum likelihood method was used to estimate parameters using LISREL 8.0 (Joreskog \& Sorbom, 1993).

The results of the standardized factor loadings for the common factor model are displayed in Fig. 1. The chi-square value of this model is 197.27 ( $\mathrm{df}=27, p<.01)$, rejecting this model. In addition, other fit indices also reveal that this common factor model is not retainable (RMSEA $=.17$, 90\% C.I. for RMSEA =.15-.18; $\mathrm{NNFI}=.92 ; \mathrm{CFI}=.94 ; \mathrm{SRMR}=.08)$. There is no improper value of parameter estimates. The squared multiple correlations for the nine indicators are ranged from .27 to .81 (see Table 2 for Model 1). Three items in the SWLS (Item 1, 3 and 4) has a value lower than 30 .

Further, the results of standardized factor loadings for the second model are displayed in Fig. 2. The chi-square value of this model is 85.12 ( $\mathrm{df}=26, p<.01)$, rejecting this model. However, most fit indices reveal that this model is retainable,

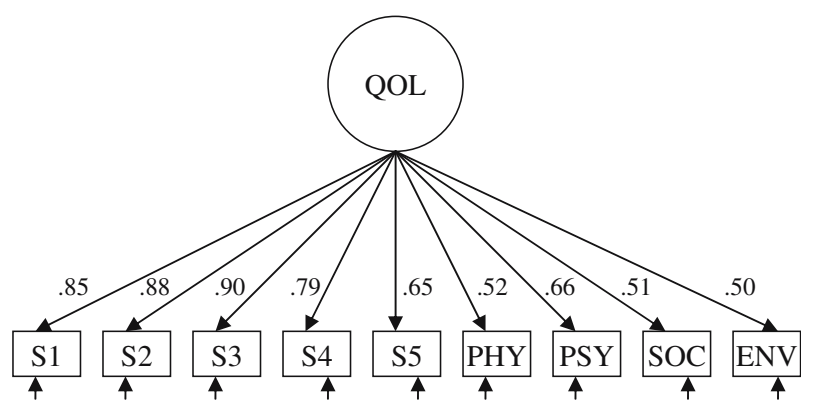

Fig. 1 Standardized estimates of factor loadings in the common factor model for scores of the SWLS and the WHOQOL-BREF. Note: S1 to S5 means the Item 1 to Item 5 in the SWLS. PHY, PSY, SOC and ENV were domain scores in the WHOQOL-BREF. All estimates were significant at $p<.05$. Estimates of uniqueness (error) were omitted for parsimony 
Table 2 Squared multiple correlations of indicators in three models

\begin{tabular}{lllc}
\hline & Model 1 & Model 2 & Model 3 \\
\hline SWLS & & & \\
Item 1 & 0.27 & 0.46 & 0.86 \\
Item 2 & 0.44 & 0.62 & 0.57 \\
Item 3 & 0.26 & 0.41 & 0.44 \\
Item 4 & 0.25 & 0.44 & 0.44 \\
Item 5 & 0.73 & 0.75 & 0.76 \\
WHOQOL-BREF & & & 0.86 \\
Physical domain & 0.78 & 0.81 & 0.80 \\
Psychological domain & 0.81 & 0.81 & 0.63 \\
Social domain & 0.63 & 0.62 & 0.51 \\
Environmental domain & 0.42 & 0.40 &
\end{tabular}

although values of fit indices are not satisfactory (RMSEA $=.09,90 \%$ C.I. for RMSEA $=.07-.11 ; \mathrm{NNFI}=.97 ; \mathrm{CFI}=.98 ;$ SRMR $=.05)$. Generally, this correlated two-factor model performed better than the first common-factor model in terms of these fit indices. The correlation between the two factors is .75. There is no improper value of parameter estimates. The squared multiple correlations for the nine indicators are ranged from .40 to .81 (see Table 2 for Model 2).

Finally, results of the standardized factor loadings for the third model are displayed in Fig. 3. The chi-square value of this model is $26.04(\mathrm{df}=17, p>.05)$, retaining this model. In addition, all fit indices also reveal that this model is excellent $(\mathrm{RMSEA}=.04,90 \%$ C.I. for RMSEA $=.00-.07$; NNFI $=.99 ; \mathrm{CFI}=1.00 ;$ SRMR $=$ $.023)$. Moreover, the result also show that there was a significant correlation between global approach and domain approach $(r=.30, p<.05)$. There is no improper value of parameter estimates. The squared multiple correlations for the nine indicators are ranged from .44 to .86 (see Table 2 for Model 3).

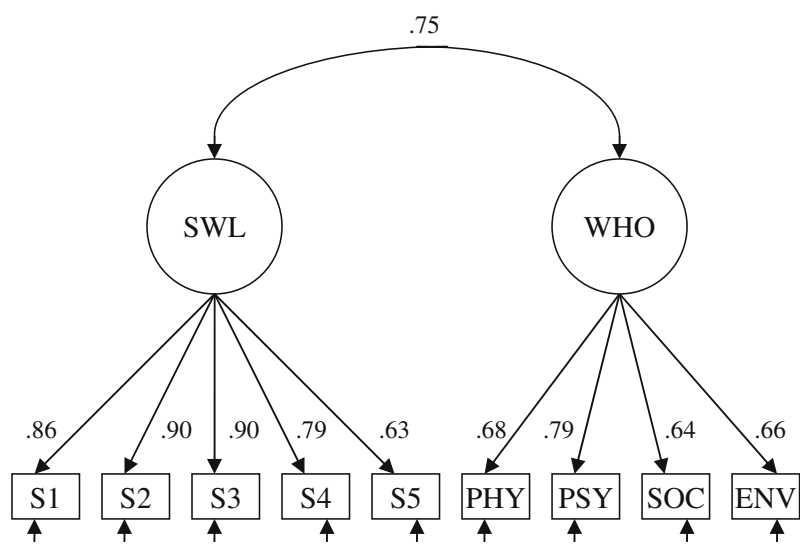

Fig. 2 Standardized estimates of factor loadings in the correlated two-factor model for scores of the SWLS and the WHOQOL-BREF. Note: S1 to S5 means the Item 1 to Item 5 in the SWLS. PHY, PSY, SOC and ENV were domain scores in the WHOQOL-BREF. All estimates were significant at $p<.05$. Estimates of uniqueness (error) were omitted for parsimony 


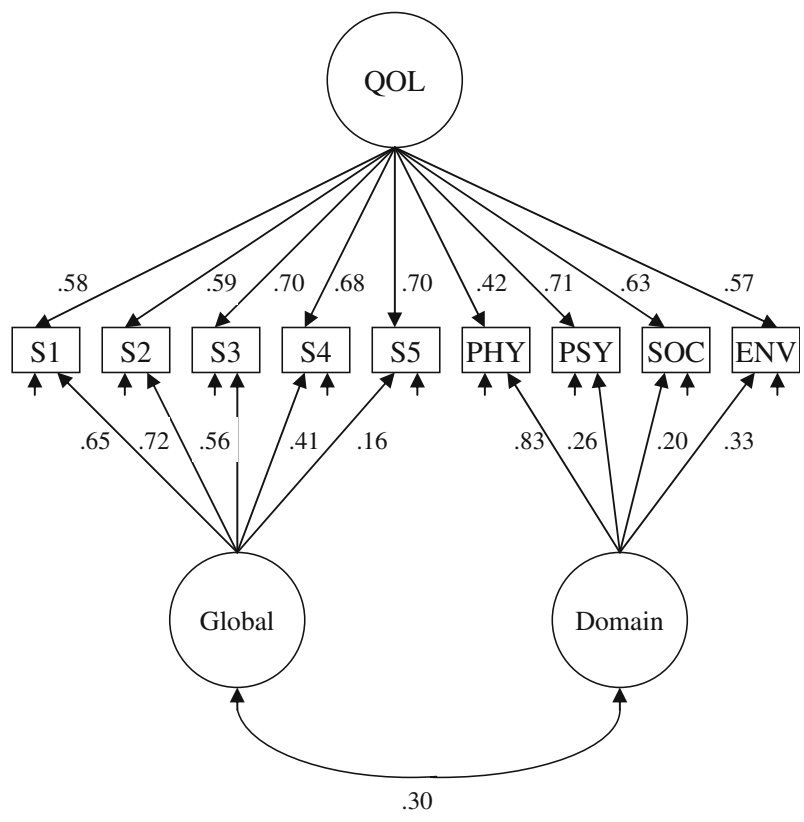

Fig. 3 Standardized estimates of factor loadings in the three-factor model for scores of the SWLS and the WHOQOL-BREF. Note: S1 to S5 means the Item 1 to Item 5 in the SWLS. PHY, PSY, SOC and ENV were domain scores in the WHOQOL-BREF. All estimates were significant at $p<.05$. Estimates of uniqueness (error) were omitted for parsimony

Among the three models, the third model is superior to the first two models on the fit indices, squared multiple correlations of each indicator reported above. Moreover, the fit indices developed for model comparison, AIC, CAIC and ECVI (lower value means a better model), also reveal that the third model is better than the other two models. The AIC values from the first model to the third model are $287.45,128.92$ and 81.62 , respectively. The CAIC values from the first model to the third model are 372.36, 218.54 and 213.70 , respectively. The ECVI values from the first model to the third model are $.95, .43$ and .27 , respectively. The third model has the lowest values on AIC, CAIC and ECVI. Thus, these two indices also support the finding that the third model is better than the first two.

\section{Discussion}

The purpose of this study was to examine the structure of global and domain measurements on quality of life from the psychometric perspective. Three CFA models were specified and compared. The results show that the three-factor model in which one common factor for quality of life and two factors for global and domain approaches is the best model. This model reveals that the SWLS and the WHOQOL-BREF assessed the same construct on quality of life and also shows that global and domain approaches impact measurements.

This argument will be more cogent if we compare the three-factor model with the common factor model and the correlated two-factor model. First, when comparing 
the common factor model with the three-factor model, it can be found that it is not appropriate to assume that global and domain measures have the same meaning as specified in the common factor model. Second, when comparing the correlated twofactor model with the three-factor model, it can be found that treating the global and domain measures as two distinct but related constructs is also inappropriate. Therefore, comparisons of these three models in this study suggest that (1) global and domain measures on quality of life do not have exactly the same meaning; however, (2) they do assess the same construct on quality of life; and (3) global and domain approaches do impact scores.

Although there are debates on model evaluation of structural equation modeling, for example, (1) should we totally rely on chi-square test of exact fit or approximate fit indices for model evaluation (Barrett, in press; Goffin, in press; Markland, in press; McIntosh, in press)? (2) is there any golden rule in the usage of approximate fit indices (Beauducel \& Wittmann, 2005; Fan \& Sivo, 2005; Marsh et al., 2004; Yuan, 2005)?, etc, these debates would not change the conclusion of this study. Specifically, if we only consider the results of chi-square test, then it can be found that only the third model is retained. Both first and second model are rejected with its corresponding degree of freedoms. If we only consider the results of fit indices, it can be also found that the third model has highest values on relative indices (CFI, NNFI), and lowest values on absolute indices (RMSEA, SRMR). The performances of these fit indices of the third model are even better than the criteria set by $\mathrm{Hu}$ and Bentler (1999). Moreover, model comparison from AIC, CAIC and ECVI also reveal that the third model is better than the first two models. For the accounted variance of each indicator, results of squared multiple correlations show that the third model is better than the first two models. Therefore, all these model evaluation information converges to the same conclusion.

However, there are some limitations of this study. First, participants in this study are university students. It is not a random sample for research. It is desirable to see if there is any national survey data, which include global and domain-specific measures of QOL and use the data to cross-validate the finding of the current study. Second, from modeling perspective, the indicators of the SWLS and the WHOQOL-BREF are not assessed at the same level. That is, the indicators of the SWLS are the item scores, which are ordinal data in the nature, while the indicators of the WHOQOLBREF are the four domain scores, which are scores summated from several item scores. This situation would not result in a major problem for modeling in the current study, because there is no serious non-normality problem among these indicators. However, it would be result in asymmetry information taken from the SWLS and the WHOQOL-BREF. Specifically, the current study takes more item information for the SWLS than the WHOQOL-BREF. This situation may lead us to take trivial characteristics of an item (such as an effect result from specific wording) of the SWLS in modeling.

For example, the first three items in the SWLS are regarded as items for life satisfaction at present condition (In most ways my life is close to my ideal; The conditions of my life are excellent; I am satisfied with my life) and the last two items are treated as items for life satisfaction introspected from past experiences (So far I have gotten the important things I want in life; If I could live my life over, I would change almost nothing) (McDonald, 1999; Wu \& Yao, 2006c). This wording characteristic of the SWLS do reflect on the estimates of factor loadings. Previous studies on psychometric properties of the SWLS have found that factor loadings of the last 
two items usually lower than the first three items (McDonald, 1999; Pavot \& Diener, 1993; Wu \& Yao, 2006c). Similar pattern is also found in the three models in the current study. Factor loading of the last two items are lower than the first three items on (1) the general factor in Model 1, (2) the SWLS factor in Model 2, and (3) the global approach factor in Model 3. This result shows that the specific wording of items in the SWLS is accounted in the model. In order to avoid including trivial characteristics of an item, it is better adding more global life satisfaction measurements to compute several summated scores as the indicators for global QOL in future studies.

Finally, we only model the relationship between global and domain-specific measures of QOL using only two measurements in QOL literature (the SWLS and the WHOQOL-BREF); it cannot be sure that if the results can be hold when different measurements are used. For example, in the three-factor model used in the current study, it is found that there is a low but significant correlation between the global and domain approaches, revealing that global and domain approaches are not independent constructs. This finding may reflect the result observed in Lance et al.'s (1995) study, in which they found a non-recursive relationship between global measure and domain-specific measures. Nonetheless, further investigation is needed to cross-validate this finding because only one sample and two measurements (the SWLS and the WHOQOL-BREF) were used in the current study, and the correlation is not substantially large enough to make a strong conclusion.

Generally, the current finding is consistent with Davis, Morris, and Kraus' (1998) findings concerning global and domain measurement issues on social support. In their study, they investigated the relationship between global and relationship-specific (domain) perceptions of social support by measures of global support, domain support from four significant others, global well-being, and domain well-being. They used hierarchical regression analysis to show that global support has a significant effect on measures of global well-being after controlling for domain supports, and domain supports had significant effects on domain well-being after controlling for global supports. Accordingly, they concluded that social support measured by global and domain approaches seems to be two distinct constructs. Although Davis et al. (1998) used regression analysis rather than confirmatory factor analysis to analyze their data, their results show that global and domain measures of social support and well-being have different performances, and these differences can be interpreted by considering the measurement approaches.

In future studies, the three-factor model could be examined more thoroughly by using different research designs to see if global and domain approaches represent stable response characteristics. For example, the three-factor model might be examined by incorporating other global and domain measures of quality of life or other constructs, such as social support mentioned above, to determine if there is a stable global approach effect underlying all global measures and a stable domain approach effect underlying all domain measures. Moreover, the model could also be examined with longitudinal design to investigate if global and domain approaches represent stable characteristics across time.

After ensuring that global and domain approaches represent stable response characteristics we should ask what characteristics they represent. If we take the current results into account, it would be suspected that there is a common response mechanism underlying responses on global measurements and a common response mechanism underlying responses on domain measurements. Although we cannot 
provide solid evidence here to clarify the two mechanisms underlying responses on global measurements and domain measurements, a possible research direction can be derived from cognitive psychology to see if there are two different mental operations individuals use when responding to global measurements and domain measurements.

For example, one possible research idea can be derived from the global-domain issue in the cognitive process of graph comprehension. Ratwani, Trafton, and Boehm-Davis (2003) conducted a study to examine if there are qualitative differences between the way people answer global and local questions and how different questions activate various cognitive operations on graph comprehension. In their first study, the results showed that global questions, which required general trends or descriptions to be identified, and local questions, which required straightforward single extractions from the graph, elicit different cognitive operations by participants. For global questions, participants extracted primarily aggregate information, while they extracted primarily local information when answering local questions. In their second study, eye-track data further showed that participants inspected graphs by different steps when answering local questions verses global questions. Their findings suggested that cognitive processes for local questions and global questions are different. Although the results from Ratwani, Trafton, and Boehm-Davis' research on graph comprehension cannot be directly applied to quality of life measurement addressed here, their study provides insight into the mechanisms underlying the responses on global and domain measurements of quality of life from the cognitive perspective.

In other words, in future studies, researchers can examine responses to global and domain measurements of quality of life to ascertain if they are derived from different cognitive mechanisms, for example, responses to global measurements of quality of life may be related to more abstract concepts, and responses to domain measurements of quality of life may be related to more concrete experiences of that domain. One can use response time as an index to see if global evaluation has quick link with abstract concepts than concrete experiences, and if domain evaluation has quick link with concrete experiences than abstract concepts. Examining this hypothesis can facilitate us to know if participants have different thinking style when they face questions for global and domain evaluations. Of course, this is just a simple idea in investigating the cognitive mechanisms of global and domain evaluations. There are many challenging task waiting for us to figure out the cognitive mechanisms of global and domain evaluations. However, investigating cognitive mechanisms of global and domain evaluations is a promising pathway to understanding the nature of global and domain evaluations.

Acknowledgements This study was supported by the National Science Council (NSC 94-2413-H002-018) and the National Health Research Institute (NHRI-EX94-9204PP).

\section{References}

Arrindell, W. A., Heesink, J., \& Feij, J. A. (1999). The Satisfaction with Life Scale (SWLS): Appraisal with 1700 health young adults in the Netherlands. Personality and Individual Differences, 26, 815-826. 
Arrindell, W. A., Meeuwesen, L., \& Huyse, F. J. (1991). The Satisfaction with Life Scale (SWLS): Psychometric properties in a non-psychiatric medical outpatients sample. Personality and Individual Differences, 12, 117-123.

Atienza, F. L., Balaguer, I., \& Garcia-Merita, M. L. (2003). Satisfaction with Life Scale: Analysis of factorial invariance across sexes. Personality and Individual Differences, 35, 1255-1260.

Barrett, P. Structural equation modeling: Adjudging model fit. Personality and Individual Differences (in press).

Beauducel, A., \& Wittmann, W. (2005). Simulation study on fit indexes in confirmatory factor analysis based on data with slightly distorted simple structure. Structural Equation Modeling, 12, 41-75.

Bentler, P. M. (1990). Comparative fit indexes in structural models. Psychological Bulletin, 107, 238246.

Browne, M. W., \& Cudeck, R. (1993). Alternative ways of assessing model fit. In K. A. Bollen \& J. S. Long (Eds.), Testing structural equation models. Beverly Hills, CA: Sage.

Cohen, E. H. (2000). A facet theory approach to examining overall and life facetsatisfaction relationships. Social Indicators Research, 51, 223-237.

Davis, M. H., Morris, M. M., \& Kraus, L. A. (1998). Relationship-specific and globalperceptions of social support: Associations with well-being and attachment. Journal of Personality and Social Psychology, 74, 468-481.

Diener, E. (1984). Subjective well-being. Psychological Bulletin, 95, 542-575.

Diener, E., Emmons, R. A., Larsen, R. J., \& Griffin, S. (1985). The Satisfaction with Life Scale. Journal of Personality Assessment, 49, 71-75.

Diener, E., Suh, E. M., Lucas, R., \& Smith, H. (1999). Subjective well-being: Three decades of progress. Psychological Bulletin, 125, 276-302.

Fan, X., \& Sivo, S. A. (2005). Sensitivity of fit indexes to misspecified structural or measurement model components: Rationale of two-index strategy revisited. Structural Equation Modeling, 12, 343-367.

Goffin, R. D. Assessing the adequacy of structural equation models: Golden rules and editorial policies. Personality and Individual Differences (in press).

Hoyle, R. H. (1995). The structural equation modeling approach: Basic concepts and fundamental issues. In R. H. Hoyle (Ed.), Structural equation modeling: Concepts, issues, and applications (pp. 1-15). Thousand Oaks: Sage Publications.

Hsieh, C. M. (2003). Counting importance: The case of life satisfaction and relative domain importance. Social Indicators Research, 61, 227-240.

Hsieh, C. M. (2004). To weight or not to weight: The role of domain importance in quality of life measurement. Social Indicators Research, 68, 163-174.

Hu, L., \& Bentler, P. M. (1999). Cutoff criteria for fit indexes in covariance structure analysis: Conventional criteria versus new alternatives. Structural Equation Modeling, 6, 1-55.

Joreskog, K. G., \& Sorbom, D. (1993). LISREL 8: Structural equation modeling with the SIMPLIS command language. Hillsdale, NJ: Lawrence Erlbaum Associates.

Lance, C. E., Mallard, A. G., \& Michalos, A. C. (1995). Tests of the causal directions of global-life facet satisfaction relationships. Social Indicators Research, 34, 69-92.

Lewis, C. A., Shevlin, M. E., Bunting, B. P., \& Joseph, S. (1995). Confirmatory factor analysis of the satisfaction with life scale: Replication and methodological refinement. Perceptual and Motor Skills, 80, 304-306.

Lucas, R. E., Diener, E., \& Suh, E. (1996). Discriminant validity of well-being measures. Journal of Personality and Social Psychology, 71, 616-628.

Markland, D. The golden rule is that there are no golden rules: A commentary on Paul Barrett's recommendations for reporting model fit in structural equation modeling. Personality and Individual Differences (in press).

Marsh, H. W., Hau, K. T., \& Wen, Z. (2004). In search of Golden rules: Comment on hypothesistesting approaches to setting cutoff values for fit indexes and dangers in overgeneralizing $\mathrm{Hu}$ and Bentler's (1999) findings. Structural Equation Modeling, 11, 320-341.

McDonald, R. P. (1999). Test theory: A unified treatment. Mahwah, NJ: Lawrence Erlbaum.

McIntosh, C. N. Rethinking fit assessment in structural equation modeling: A commentary and elaboration on Barrett. Personality and Individual Differences (in press).

Pavot, W., \& Diener, E. (1993). Review of the satisfaction with life scale. Psychological Assessment, 5, 164-172.

Pavot, W., Diener, E., Colvin, C. R., \& Sandvik, E. (1991). Further validation of the satisfaction evidence for the cross-method convergence of well-being. Social Indicators Research 28, 1-20. 
Ratwani, R. M., Trafton, J. G., \& Boehm-Davis, D. A. (2003). 'Thinking graphically: Extracting local and global information. In R. Alterman \& D. Kirsch (Eds.), 25th Annual Meeting of the Cognitive Science Society. Boston, MA: Erlbaum.

Russell, L. B., Hubley, A. M., Palepu, A., \& Zumbo, B. D. (2006). Does weighting capture what's important? Revisiting subjective importance weighting with a quality of life measure. Social Indicators Research, 75, 141-167.

Sachs, J. (2003). Validation of the Satisfaction with Life Scale in a sample of Hong Kong University students. Psychologia, 46, 225-234.

Shevlin, M. E., Brunsden, V., \& Miles, J. N. V. (1998). Satisfaction with Life Scale: Analysis of factorial invariance, mean structures and reliability. Personality and Individual Differences, 25, 911-916.

Shevlin, M. E., \& Bunting, B. P. (1994). Confirmatory factor analysis of the satisfaction with life scale. Perceptual and Motor Skills, 79, 1316-1318.

The WHOQOL Group (1998a). Development of the WHOQOL-BREF Quality of Life Assessment. Psychological Medicine, 28, 551-558.

The WHOQOL Group (1998b). The World Health Organization Quality of Life Assessment (WHOQOL): Development and general psychometric properties. Social Science \& Medicine, 46, $1569-1585$.

Westaway, M. S., Maritz, C., \& Golele, N. J. (2003). Empirical testing of the satisfaction with life scale: A South African pilot study. Psychological Reports, 92, 551-554.

Wu, C. H., \& Yao, G. (2006a). Do we need to weight satisfaction scores with importance ratings? Social Indicators Research, 78, 305-326.

Wu, C. H., \& Yao, G. (2006b). Do we need to weight item satisfaction by item importance? A perspective from Locke's range-of-affect hypothesis. Social Indicators Research, 79, 485-502.

Wu, C. H., \& Yao, G. (2006c). Analysis of factorial invariance across genders in the Taiwan version of the satisfaction with life scale. Personality and Individual Differences, 40, 1259-1268.

Yao, G., Chung, C. W., Yu, C. F., \& Wang, J. D. (2002). Development and verification of reliability and validity of the WHOHR-QOL-BREF Taiwan version. Journal of the Formosan Medical Association, 101, 342-351.

Yuan, K. H. (2005). Fit indices versus test statistics. Multivariate Behavioral Research, 40, 115-148. 